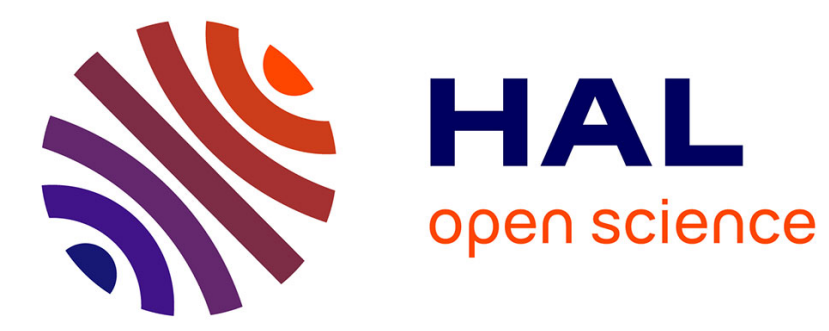

\title{
MAGNETIC ANISOTROPY IN THE Fe-BORACITES
}

R. Link, W. Wurtinger

\section{To cite this version:}

R. Link, W. Wurtinger. MAGNETIC ANISOTROPY IN THE Fe-BORACITES. Journal de Physique Colloques, 1974, 35 (C6), pp.C6-581-C6-587. 10.1051/jphyscol:19746125 . jpa-00215738

\section{HAL Id: jpa-00215738 https://hal.science/jpa-00215738}

Submitted on 1 Jan 1974

HAL is a multi-disciplinary open access archive for the deposit and dissemination of scientific research documents, whether they are published or not. The documents may come from teaching and research institutions in France or abroad, or from public or private research centers.
L'archive ouverte pluridisciplinaire HAL, est destinée au dépôt et à la diffusion de documents scientifiques de niveau recherche, publiés ou non, émanant des établissements d'enseignement et de recherche français ou étrangers, des laboratoires publics ou privés. 


\title{
MAGNETIC ANISOTROPY IN THE Fe-BORACITES
}

\author{
R. LINK and W. WURTINGER
}

Institut für Kernphysik, Technische Hochschule Darmstadt, West Germany

\begin{abstract}
Résumé. - Nous avons étudié à l'aide de la technique Mössbauer les interactions hyperfines électrique et magnétique dans les boracites de fer $\mathrm{Fe}_{3} \mathrm{~B}_{7} \mathrm{O}_{13} \mathrm{X}(\mathrm{X}=\mathrm{Cl}, \mathrm{Br}, \mathrm{I})$. L'analyse des spectres Mössbauer en dessous de la température de Néel révèle 3 sites cristallographiques dans le cas des boracites $\mathrm{Fe}-\mathrm{Cl}$ et $\mathrm{Fe}-\mathrm{Br}$ et au moins 4 sites dans la boracite $\mathrm{Fe}-\mathrm{I}$. Ces sites diffèrent par les valeurs du champ magnétique hyperfin et leurs orientations par rapport au tenseur gradient de champ électrique. La température de Néel s'est révélée être $33,2 \pm 0,2 \mathrm{~K}$ et $11,8 \pm 0,2 \mathrm{~K}$ pour les boracites $\mathrm{Fe}-\mathrm{I}$ et $\mathrm{Fe}-\mathrm{Cl}$ respectivement.

Les valeurs expérimentales des champs hyperfins sont comparées à des calculs basés sur la théorie du champ des ligands incluant le couplage spin-orbite et un champ moléculaire. A l'aide de ce modèle on peut expliquer l'importante contribution orbitale au champ hyperfin et l'anisotropie magnétique.
\end{abstract}

\begin{abstract}
Using the Mössbauer technique we have investigated the electric and magnetic hyperfine interaction in Fe-boracites $\mathrm{Fe}_{3} \mathrm{~B}_{7} \mathrm{O}_{13} \mathrm{X}(\mathrm{X}=\mathrm{Cl}, \mathrm{Br}, \mathrm{I})$. The analysis of the Mössbauer spectra below the Néel temperature leads to 3 lattice sites in the case of $\mathrm{Fe}-\mathrm{Cl}$ - and $\mathrm{Fe}-\mathrm{Br}$-boracite and to at least 4 sites in the Fe-I-boracite, which are different with regard to the sizes of the magnetic hyperfine fields and their directions with respect to the electric field gradient tensor. The Néel temperature turned out to be $33.2 \pm 0.2 \mathrm{~K}$ and $11.8 \pm 0.2 \mathrm{~K}$ for the Fe-I- and Fe-Cl-boracite, respectively.

The experimental values for the hyperfine fields are compared with calculations based on Ligand Field Theory including spin orbit coupling and molecular field approach. Within this model the strong orbital contribution to the hyperfine field and the magnetic anisotropy can be explained.
\end{abstract}

1. Introduction. - Recently much attention has been paid to the compound boracite $\mathrm{M}_{3} \mathrm{~B}_{7} \mathrm{O}_{13} \mathrm{X}$, where $\mathrm{M}$ stands for a divalent metal ion and $\mathrm{X}$ for one of the halogens $\mathrm{Cl}, \mathrm{Br}$ or $\mathrm{I}$. These boracites have nearly the same crystal structure as the natural boracite $\mathrm{Mg}_{3} \mathrm{~B}_{7} \mathrm{O}_{13} \mathrm{Cl}[1,2,3]$. The crystal structure is orthorhombic below and cubic above the ferroelectric Curie Temperature. The existence of ferroelectricity in the orthorhombic structure is a characteristic property of all boracite. Mössbauer measurements on iron boracite by J. M. Trooster [4] showed a further phase transition from orthorhombic to trigonal structure.

The magnetic properties of the $3 \mathrm{~d}$-metal boracites have been investigated by measuring the susceptibility $[5,6]$. It turned out that the boracites show antiferromagnetic ordering at low temperatures in the ferroelectric phase. The antiferromagnetism is accompanied by a weak ferromagnetism.

Recently Mössbauer measurements on Fe-Br-boracite in the magnetically ordered phase have been published by R. Jagannathan et al. [11]. It was the aim of these Mössbauer measurements to investigate the hyperfine fields at the ${ }^{57} \mathrm{Fe}$ nucleus in all three ironboracites below the Néel-temperature.

2. Experimental arrangement. - The Mössbauer spectra of the $\mathrm{Fe}-\mathrm{Cl}-, \mathrm{Fe}-\mathrm{Br}-$ and $\mathrm{Fe}-\mathrm{I}$-boracite have been taken in transmission geometry using a variable temperature He-flow cryostat [7]. The control and measurement of the temperature of the source and the absorber was performed by measuring the resistance of Allan-Bradley resistors.

The details of the Mössbauer spectrometer have been described elsewhere [8]. The velocity was changed periodically in a triangular way using a Multi Channel Analyzer in the Multiscaling mode. The $10 \mathrm{mCi}$ single line source consisted of ${ }^{57} \mathrm{Co}$ in a copper matrix. Polycristalline material was used as absorbers (Fe-Cl- : $20 \mathrm{mg} / \mathrm{cm}^{2}$, Fe-Br- : $130 \mathrm{mg} / \mathrm{cm}^{2}$, Fe-I-boracite : $90 \mathrm{mg} / \mathrm{cm}^{2}$ ). The compounds were prepared at the «II. Physikalisches Institut der TH Darmstadt», according to a technique proposed by H. Schmid [9].

3. Analysis and results. - The spectra of the Feboracites below the Néel temperature are rather complex. Because of the strong electric quadrupole interaction in the trigonal phase, one has to assume that, with the onset of the magnetic ordering, electric and magnetic hyperfine splittings are of the same order of magnitude.

Therefore a Least-Squares-Fitting procedure has been developed, which allowed us to fit a $\frac{3}{2}-\frac{1}{2}$ M 1 nuclear transition in the presence of electric $\left(H_{\mathrm{O}}\right)$ and magnetic $\left(H_{\mathrm{m}}\right)$ hyperfine interaction of arbitrary rela- 
tive size and arbitrary angle $(\theta, \varphi)$ between the main axis of the field gradient tensor $\left(V_{i j} ; i, j=x, y, z\right)$ and the effective magnetic field $H_{\mathrm{n}}$.

$x=H_{Q}+x_{m}$

$\mathscr{H}_{\mathrm{Q}}=\frac{\mathrm{e} Q V z z}{4 I(2 I-1)}\left(3 I_{z}^{2}-I^{2}+\frac{\eta}{2}\left(I_{+}^{2}+I_{-}^{2}\right)\right)$

$\mathscr{H}_{\mathrm{m}}=-g u_{\mathrm{N}} H_{\mathrm{n}}\left(I_{z} \cos \theta+\left(I_{x} \cos \varphi+I_{y} \sin \varphi\right) \sin \theta\right)$.

We have used the following values for the nuclear moments of the groundstate $g_{0}=0.0904206$ and excited state $g_{1}=0.15463$ and $Q=0.196$ barn [10]. The fit of the spectra showed that one had to assume three lattice sites for the $\mathrm{Fe}-\mathrm{Cl}$ - and $\mathrm{Fe}-\mathrm{Br}$-boracite, whereas four were needed in the case of the $\mathrm{Fe}-\mathrm{I}$ boracite. The large number of parameters, which had to be determined, made it necessary to correlate some parameters or to keep some parameters fixed. Thus we assumed that the field gradient, the asymmetry parameter as well as the isomer shift each possess the same values for all lattice sites. In the case of the Fe-I-boracite the line-widths of all sites have been fixed.

The strong correlation between $V_{i j}, \eta$ and $\theta, \varphi$ gives the same $\chi^{2}$ for different sets of parameters. This leads to the big errors in the parameter set. Our values and those of $\mathrm{R}$. Jagannathan et al. for the $\mathrm{Fe}-\mathrm{Br}$-boracite [11] agree within their errors. The hyperfine parameters of the best fit for $\mathrm{Fe}-\mathrm{Cl}-, \mathrm{Fe}-\mathrm{I}$ - and $\mathrm{Fe}-\mathrm{Br}$ boracite at $5 \mathrm{~K}, 8 \mathrm{~K}$ and $4.2 \mathrm{~K}$, respectively, are given in table $\mathrm{I}$.

The $\mathrm{Fe}-\mathrm{Cl}$ - and $\mathrm{Fe}$-I-boracite spectra, taken at different temperatures, are shown in figure 1 and 2 . In figure 3 we have plotted the measured reduced magnetic hyperfine fields for $\mathrm{Fe}-\mathrm{Cl}$ - and $\mathrm{Fe}-\mathrm{I}$-boracite as function of $T / T_{\mathrm{N}}$. The experimental values for the Néel temperatures are $11.8 \pm 0.2 \mathrm{~K}$ and $33.2 \pm 0.2 \mathrm{~K}$ for the $\mathrm{Fe}-\mathrm{Cl}$ - and Fe-I-boracite, respectively. The solid lines represent the curve of the Brillouin-function for
$S=2$. The deviation may result from the strong magnetic anisotropy which will be discussed in the succeeding sections.

4. Discussion. - The hyperfine interaction in the Fe-boracites has been investigated in the temperature region where the crystals order ferroelectrically and therefore the polarization must be taken into account. The discrepancy from the $S=2$ Brillouin-function in the magnetically ordered phase as well as the observed weak ferromagnetism and the measured values for the effective magnetic moment of 5.5-5.7 $\mu_{\mathrm{B}}$ [6] indicate that spin orbit coupling and possibly biquadratic exchange terms play an important role in the Hamiltonian of the $\mathrm{Fe}^{2+}$-ions.

In our calculations which are based on ligand field theory including spin orbit coupling and the molecular field model, the electric polarization was taken into account by introducing permanent electric dipole moments at the ligand sites. We used one general covalency parameter $\alpha^{2}=0.7$ [12] and assumed the exchange interaction to be isotropic.

Of course, these are rather restricting conditions and we want to point out that the results of our calculations can only be expected to be very approximate.

First we present the calculations of the electric field gradient tensor (EFG) above the Néel temperature which we used to determine the direction of the principal axis of the EFG. The calculations of the magnetic hyperfine interaction are discussed in the succeeding section.

4.1 ELECTRIC HYPERFINE INTERACTION. - The EFG results from the ligand $\left(E F G^{\text {lig }}\right)$ and from the ionic $\left(E F G^{i o n}\right)$ contribution of the $\mathrm{Fe}^{2+}$-ions. Within the frame of the ligand field model both can be calculated if the crystal structure is known. The crystal field splitting for a $d$ electron assuming point charges at the

\section{TABLE I}

Hyperfine parameter of the three iron boracites at $4,2,5$ and $8 \mathrm{~K}$ for the different lattice sites, which have been derived from the best fit of the spectra. Isomer shift with respect to the ${ }^{57} \mathrm{Co} / \mathrm{Cu}$ source $(\delta)$ and quadrupole splitting $\left(\Delta=e Q V_{z z} / 2\right)$ are given in $\mathrm{mm} / \mathrm{s}$, the effective hyperfine field $\left(H_{n}\right)$ is given in $\mathrm{kOe}$. Int. is the relative intensity.

\begin{tabular}{|c|c|c|c|c|c|c|c|c|c|}
\hline Boracite & T K & I. s. & $\delta$ & $\Delta$ & $\eta$ & $H_{n}$ & $\theta$ & $\varphi$ & Int \\
\hline & & $\overline{1}$ & $0.937(6)$ & $-2.70(3)$ & $0.97(10)$ & $147(3)$ & $21(5)$ & $0(20)$ & $1.0(1)$ \\
\hline $\mathrm{Fe}-\mathrm{Cl}$ & 5 & 2 & $0.937(6)$ & $-2.70(3)$ & $0.97(10)$ & $97(3)$ & $34(5)$ & $16(15)$ & $1.2(1)$ \\
\hline & & 3 & $0.937(6)$ & $-2.70(3)$ & $0.97(10)$ & $57(5)$ & $56(5)$ & $52(15)$ & $1.2(1)$ \\
\hline & & 1 & $0.924(6)$ & $-2.73(3)$ & $0.9(1)$ & $153(3)$ & $34(5)$ & $0(20)$ & $1.0(1)$ \\
\hline $\mathrm{Fe}-\mathrm{Br}$ & 4.2 & 2 & $0.924(6)$ & $-2.73(3)$ & $0.9(1)$ & $110(3)$ & $35(5)$ & $13(15)$ & $1.1(1)$ \\
\hline & & 3 & $0.924(6)$ & $-2.73(3)$ & $0.9(1)$ & $77(5)$ & $41(5)$ & $29(15)$ & $1.1(1)$ \\
\hline & & 1 & $0.910(6)$ & $-2.50(10)$ & $1.0(1)$ & $212(8)$ & $51(20)$ & $0(30)$ & $1.0(2)$ \\
\hline $\mathrm{Fe}-\mathrm{I}$ & 8 & 2 & $0.910(6)$ & $-2.50(10)$ & $1.0(1)$ & $202(8)$ & $74(20)$ & $0(30)$ & $1.3(2)$ \\
\hline & & 3 & $0.910(6)$ & $-2.50(10)$ & $1: 0(1)$ & $143(8)$ & $46(20)$ & $21(30)$ & $1.0(2)$ \\
\hline & & 4 & $0.910(6)$ & $-2.50(10)$ & $1.0(1)$ & $124(10)$ & $78(20)$ & $10(30)$ & $1: 0(2)$ \\
\hline
\end{tabular}



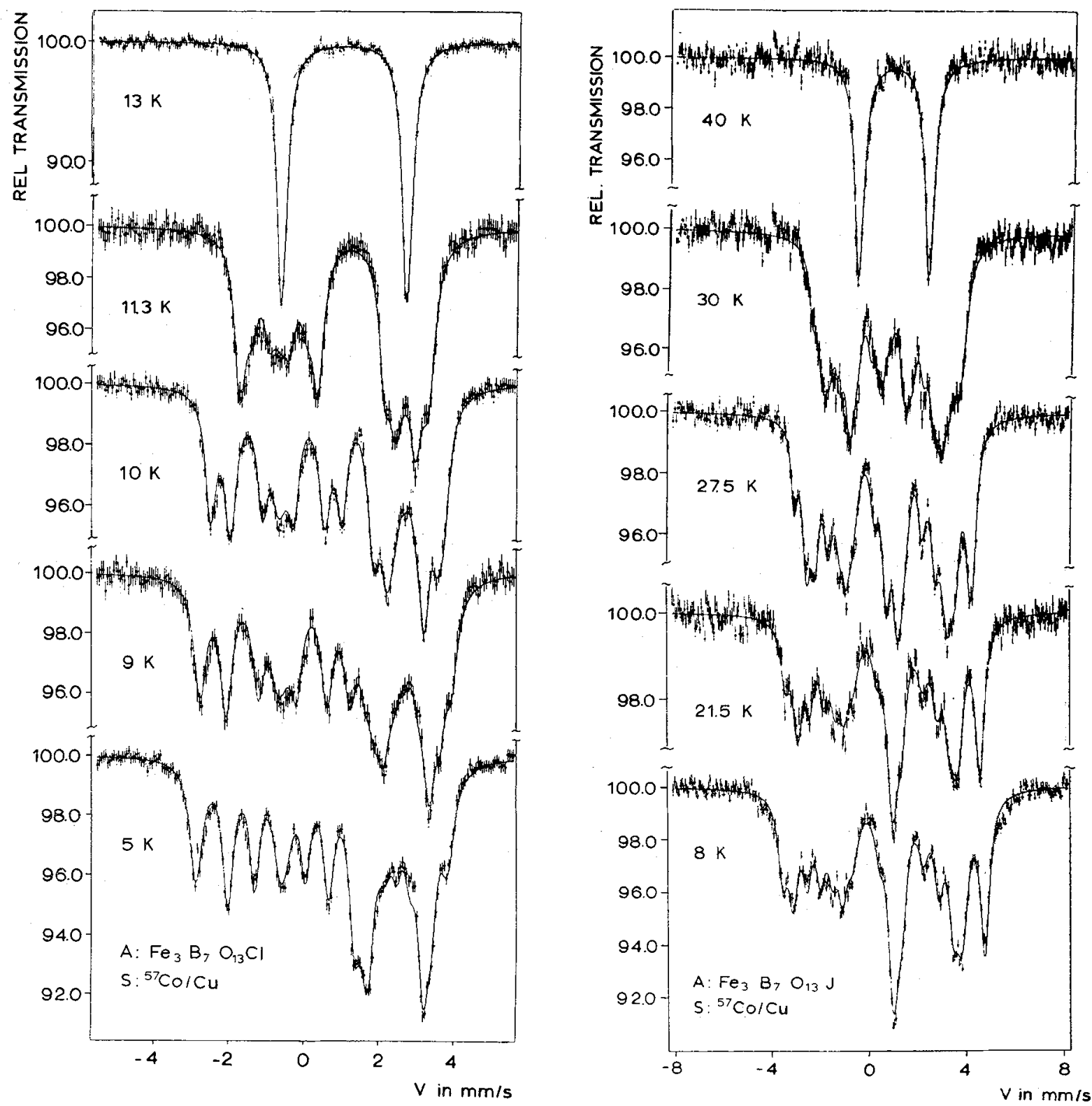

FIG. 1 and 2. - Fe-Cl- and Fe-I-boracite spectra in the temperature region between 40 and $4.2 \mathrm{~K}$. Curves drawn through the spectra represent the best fit.

six nearest neighbours of the $\mathrm{Fe}^{2+}-$ ion with $\mathrm{D}_{2 \mathrm{~d}}$ and $\mathrm{C}_{2 \mathrm{v}}$ point symmetry is shown in figure 4 . In general the calculated crystal field splitting $10 \mathrm{Dq}$ is a factor $4-5$ too small $[13,14]$ compared with measured values. Optical absorption measurements on Ni-boracites of $\mathrm{E}$. Dormann [14] clearly show that the ratio of tetragonal to cubic part of the crystal field splitting is only little affected by covalency effects. Therefore only the three low lying crystal field states $A_{1}, A_{2}, B_{2}\left(C_{2 y}\right.$-symmetry) are of relevance.

The ionic contribution to the EFG can be calculated with this assumption by introducing an effective Hamiltonian $\mathfrak{H}_{\text {eff. }}$. This has been done by J. M. Trooster [4] for the Fe-boracites in the cubic phase with point symmetry $D_{2 d}$. In the trigonal phase with nearly point symmetry $C_{2 v}$, the $E$ term is split into the $A_{2}$ and $\mathbf{B}_{2}$ states. The effective Hamiltonian can then be written :

$$
\mathfrak{H}_{\text {eff }}=\Omega\left(L_{\zeta}^{2}-1\right)+\frac{\omega}{3}\left(L_{\xi}^{2}-L_{\eta}^{2}\right)+\lambda \mathbf{L} . \mathbf{S} .
$$

Here, the parameters $\Omega$ and $\omega$ are defined by

$$
2 \omega=E_{\mathbf{B}_{2}}-E_{\mathrm{A}_{2}}, \quad \Omega=E_{\mathrm{A}_{2}}-E_{\mathrm{A}_{1}}+\omega
$$

and $\xi, \eta, \zeta$ are the coordinates of the intrinsic system of the octahedron.

The ionic contribution is the thermal expectation value of

$$
\left\langle V_{z z}>=-4 e \sqrt{\pi / 5}\left\langle r^{-3}>_{3 \mathrm{~d}}<Y_{20}>\right.\right.
$$



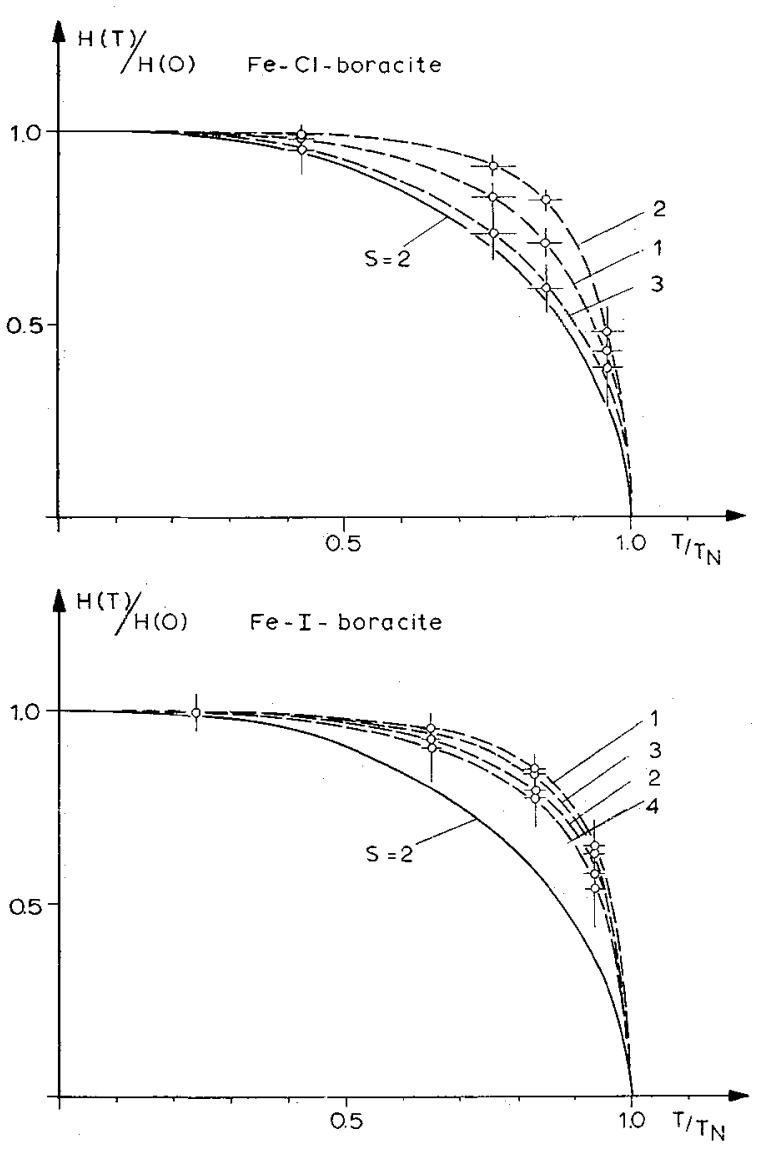

Fig. 3. - Reduced magnetic hyperfine fields of $\mathrm{Fe}-\mathrm{Cl}$ - and Fe-I-boracite for all lattice sites as function of $T / T_{\mathrm{N}}$. The values for $T_{\mathrm{N}}$ are $11.8 \pm 0.2 \mathrm{~K}$ and $33.2 \pm 0.2 \mathrm{~K}$ for $\mathrm{Fe}-\mathrm{Cl}$ - and Fe-I-boracite respectively.

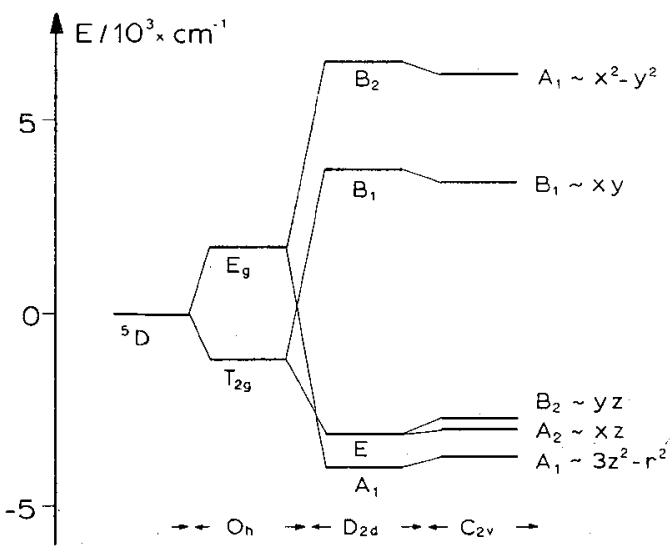

Fig. 4. - Crystal field splitting of 5D ground state for the $\mathrm{Fe}^{2+}$-ion in the case of $\mathrm{D}_{2 \mathrm{~d}}$ and $\mathrm{C}_{2 \mathrm{v}}$ point symmetry calculated on the basis of the point charge model including six nearest neighbours.

in the states $\mathrm{A}_{1}, \mathrm{~A}_{2}, \mathrm{~B}_{2}$ and therefore a function of $\Omega$, $\omega$ and $\lambda$. The temperature dependence and size of the measured field gradient in the trigonal and orthorhombic crystal phases clearly show that there is a strong contribution from the electric polarization. The crystal field potential $V(r, \vartheta, \varphi)$ at the $\mathrm{Fe}^{2+}$-ion can be expanded using the real spherical harmonics $Z_{\lambda k}$. For the $3 d^{6}$ electron configuration one gets :

$$
V(r, \vartheta, \varphi)=\sum_{\lambda=0}^{4} \sum_{\kappa=-\lambda}^{+\lambda} r^{\lambda} \gamma_{\lambda \kappa} Z_{\lambda \kappa}(\vartheta, \varphi)
$$

with

$$
\gamma_{\lambda \kappa}=\frac{4 \pi}{2 \lambda+1} \int \frac{\rho(\mathbf{R})}{R^{\lambda+1}} Z_{\lambda \kappa}(\theta, \phi) \mathrm{d} \tau_{\mathbf{R}} .
$$

Expanding $\gamma_{\lambda \kappa}$ at $\mathbf{R}_{i}$ up to the dipole term yields

$$
\begin{aligned}
\gamma_{\lambda \kappa}= & \frac{4 \pi}{2 \lambda+1} \sum_{i=1}^{N}\left\{\frac{q_{i}}{R_{i}^{\lambda+1}} Z_{\lambda \kappa}\left(\theta_{i}, \phi_{i}\right)+\right. \\
& \left.\quad+\mathbf{p}_{i}\left[\operatorname{grad}\left(\frac{Z_{\lambda \kappa}}{R^{\lambda+1}}\right)\right]_{i}+\cdots\right\} \\
= & \gamma_{\lambda \kappa}^{\mathrm{lig}}+\gamma_{\lambda \kappa}^{\mathrm{dip}} .
\end{aligned}
$$

$N$ is the number of ligands, $q_{i}$ the charge and $\mathbf{p}_{i}$ the dipole moment of the ith ion. Including the Sternheimer factors $\left(R=0.58, \gamma^{\infty}=9\right.$ for $\left.\mathrm{Fe}^{2+}[15]\right)$ one obtains for the EFG :

$\mathrm{EFG}=(1-R) \quad \mathrm{EFG}^{\mathrm{ion}}+\left(1-\gamma^{\infty}\right) \quad\left[\mathrm{EFG}^{\mathrm{lig}}+\mathrm{EFG}^{\mathrm{dip}}\right]$.

It is evident that the quadrupole splitting is a function of the crystal field parameters $\Omega, \omega$ of the spin orbit coupling constant $\lambda$ and of the electric dipole moments $\mathbf{p}_{i}$ of the ligands.

It turned out that the temperature dependence of the

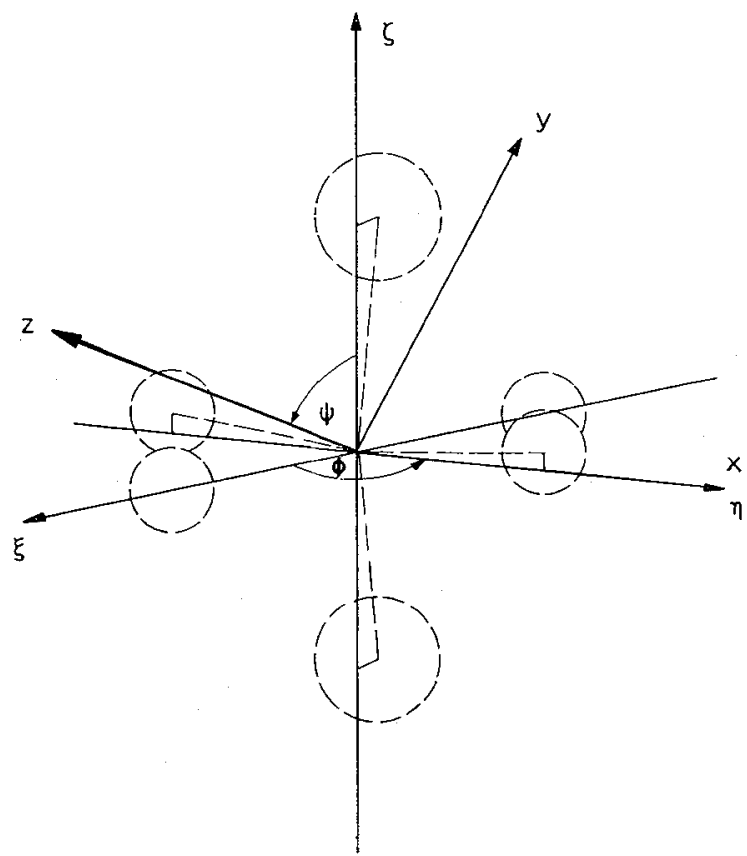

Fig. 5. - Position of the principal axis $(x, y, z)$ of the EFG with respect to the intrinsic system $(\xi, \eta, \zeta)$ in the trigonal phase. $\psi \approx 64^{\circ}, \phi=90^{\circ}$; big circles : halogen ions, small circles : oxygen ions. 
quadrupole splitting is rather insensitive with respect to the parameters $\Omega, \omega$ in the range of $\left(100-1000 \mathrm{~cm}^{-1}\right)$ and almost completely determined by the temperature dependence of $\mathbf{p}_{i}$ which we assumed to be proportional to the electric polarization pointing in the [111] direction. The electric field gradient tensor (EFG) which results from the ionic and lattice contribution which are of opposite sign and almost of equal size, is nearly diagonal in the intrinsic octahedral system. Therefore the principal axis of the EFG is mainly determined by the contribution of the dipole moments. The direction of the principal axis of the EFG with respect to the intrinsic system $(\xi, \eta, \zeta)$ which was derived by adjusting the parameters of the model to the measured quadrupole splitting is shown in figure 5 .

4.2 MAGNETIC HYPERFINE INTERACTION. - The different magnetic lattice sites, occurring in the Mössbauerspectra with the onset of the magnetic ordering, result from the spin structure and from the magnetic anisotropy of the boracites. Size and direction of the effective magnetic hyperfine field at the $\mathrm{Fe}$ nucleus will be discussed on the basis of the Ligand Field and Molecular Field Model.

Assuming the direction of the spins of a ferromagnetic sublattice to be parallel to the molecular field $\mathbf{H}_{M}$ one knows the direction of $\mathbf{H}_{M}$ once the spin structure is measured. This was done by W. v. Watburg [16] for the $\mathrm{Ni}-\mathrm{I}$-boracite using neutron scattering experiments. The spin structure is shown in figure 6 . In our calculations we assumed the spin structure to be the same for the Fe-boracites under investigation here. One obtains six different lattice sites (labeled from I-VI in Fig. 6) which differ in direction of the molecular field with respect to the intrinsic axis $(\xi, \eta, \zeta)$ of the Fe-ion.

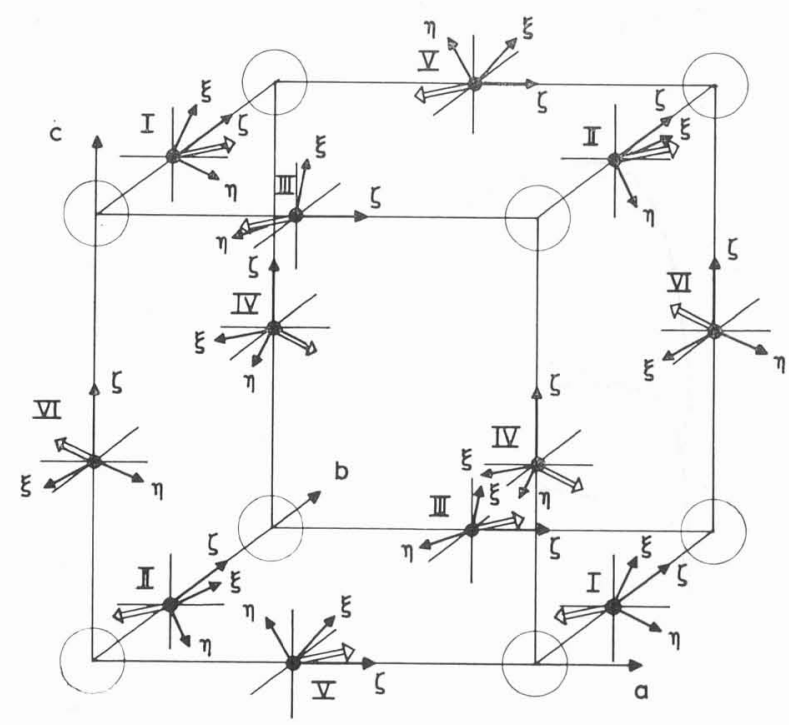

FIG. 6. - Position of the intrinsic coordinate system $(\xi, \eta, \zeta)$ of the octahedral in the cubic phase relative to the cubic cell. The directions of the spins of the Fe-ion are indicated by the arrows. Black circles : Fe-ions.
We have calculated the effective magnetic hyperfine field at the ${ }^{57} \mathrm{Fe}$ nucleus as function of the crystal field parameters $\Omega, \omega$ and of the spin orbit coupling constant $\lambda$.

Following Abragam [17]

$$
\begin{gathered}
\mathcal{H}_{\mathrm{m}}=P\{\mathbf{L I}-x \mathbf{S I}+ \\
\left.+\xi\left[L(L+1) \mathbf{S I}-\frac{3}{2}\{(\mathbf{L S})(\mathbf{L I})-(\mathbf{L I})(\mathbf{L S})\}\right]\right\} \\
P=2 g_{\mathrm{N}} \mu_{\mathrm{N}} \mu_{\mathrm{B}}<r^{-3}>_{3 \mathrm{~d}}, \quad x=-\frac{2}{3} \frac{\chi}{\left\langle r^{-3}\right\rangle_{3 \mathrm{~d}}}, \\
\xi=\frac{(2 l+1)-4 S}{S(2 l-1)(2 l+3)(2 L-1)} \\
l=L=2, \quad S=2 \\
{\left[<r^{-3}>_{3 \mathrm{~d}}=4.8 \text { a. u., } \quad \chi=-3.29\right][17,18]}
\end{gathered}
$$

After some transformations one gets the components of the effective hyperfine field operator $H_{n}^{i}(i=\xi, \eta, \zeta)$

$$
\begin{aligned}
H_{n}^{i}=- & \frac{P}{\mu_{\mathrm{N}} g_{\mathrm{N}}}\left\{L_{i}-x S_{i}+\right. \\
& \left.+\xi\left[L(L+1) S_{i}-\frac{3}{2} \sum_{j=1}^{3}\left(L_{j} L_{i}+L_{i} L_{j}\right) S_{j}\right]\right\} .
\end{aligned}
$$

The hyperfine field is the expectation value of the operator $H_{n}^{i}$, calculated with the eigenvectors of the electronic states $\left|\psi_{j}\right\rangle$

$$
\left.<H_{n}^{i}\right\rangle=\frac{\sum_{j=1}^{n}<\psi_{j}\left|H_{n}^{i}\right| \psi_{j}>\mathrm{e}^{-\Delta E_{j 0} / k T}}{\sum_{j=1}^{n} \mathrm{e}^{-\Delta E_{j 0} / k T}}
$$

The $\left|\psi_{j}\right\rangle=\sum_{K M} a_{K M}^{j}\left|\varphi_{K}\right\rangle \mid S M>$ are the linear combinations of the basis vector set $\left|\varphi_{K}\right\rangle|S M\rangle$. The $a_{K M}^{j}$ can be obtained by diagonalization of the Hamiltonian $\mathscr{H}_{\mathrm{eff}}^{\prime}$ in the 15 dimensional space of the basis vectors, where the $\left|\varphi_{K}\right\rangle(K=1,2,3)$ are symmetry adopted orbital eigenfunctions and the $|S M\rangle$ are eigenvectors of the total spin of the ion.

$$
\begin{aligned}
\mathscr{H}_{\text {eff }}^{\prime}=\Omega\left(L_{\zeta}^{2}-1\right)+\frac{\omega}{3}\left(L_{\zeta}^{2}-L_{\eta}^{2}\right)+ \\
\cdot \lambda \mathbf{L} \cdot \mathbf{S}+2 \mu_{\mathrm{B}} \mathbf{H}_{\mathrm{M}} \cdot \mathbf{S} .
\end{aligned}
$$

The expression $2 \mu_{\mathrm{B}} \quad \mathbf{H}_{\mathrm{M}} \cdot \mathbf{S}$ in $\mathcal{H}_{\text {eff }}^{\prime}$ describes the exchange interaction of the ion spins in the magnetically ordered phase, which has been approximated by the molecular field $\mathbf{H}_{\mathrm{M}}$.

In figure 7 are plotted the magnetic hyperfine field $H_{n}$ at $4.2 \mathrm{~K}$ and the angle $\theta, \varphi$ of the field with respect to the principal axis of the EFG as function of $\kappa=\Omega / \lambda$ for the six lattice sites. The parameter $\omega$ was set to $\omega=0,-100 \mathrm{~cm}^{-1}$ and $-500 \mathrm{~cm}^{-1}$. The molecular field $H_{\mathrm{M}}$ was estimated to be $30 \mathrm{kOe}$. The calculations show that the magnetic hyperfine fields coincide in size and direction for those sites where the molecular fields 


\section{C6-586}
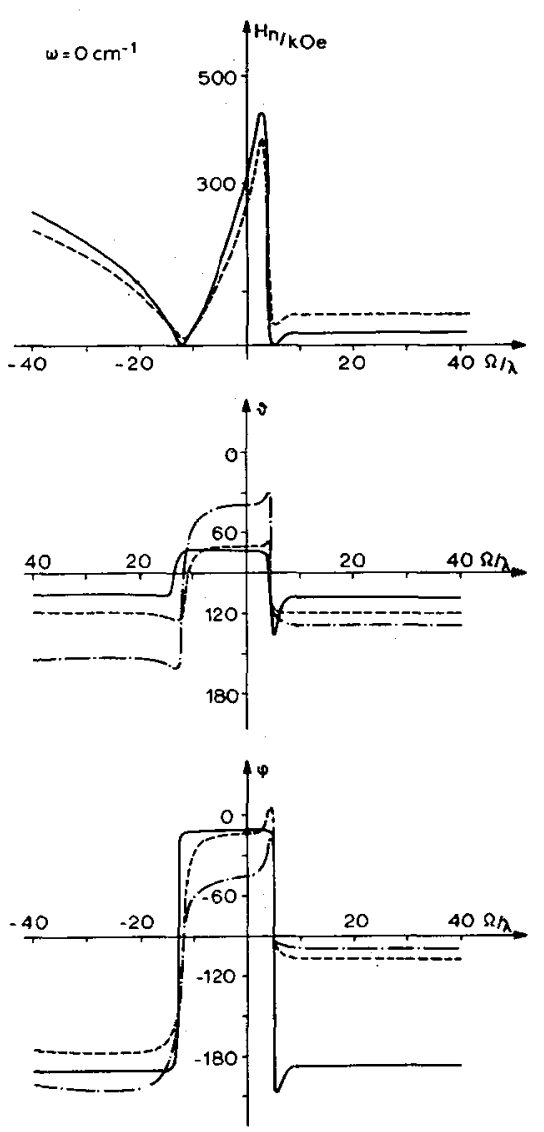
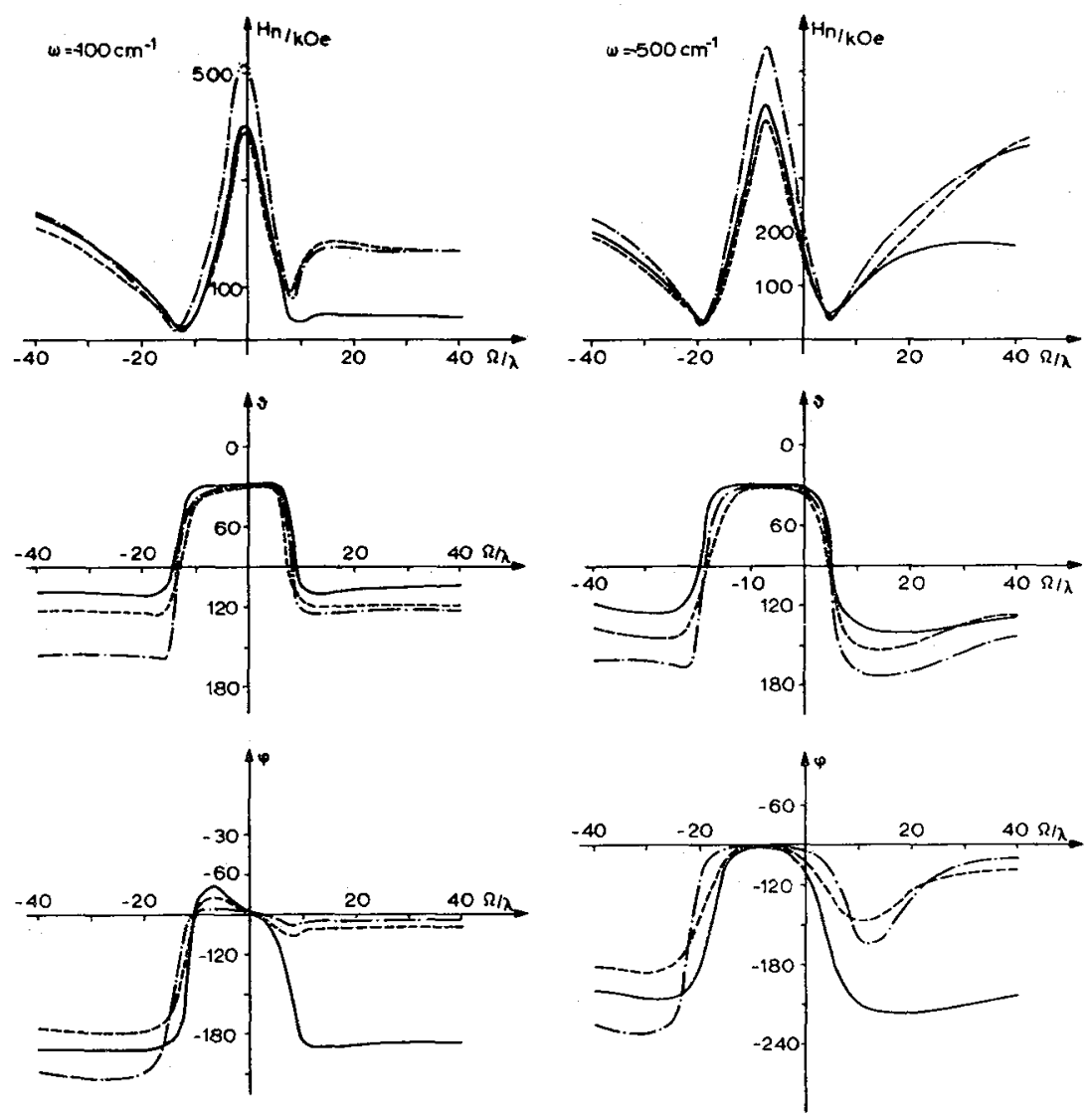

Fig. 7. - Absolute values of the hyperfine fields at the nucleus of the Fe-ions I-VI (-.. I, III ; —- II, V ; - I IV, VI) and the angles $\theta, \varphi$ with respect to the principal axis of the EFG as function of $\Omega / \lambda$ for $\omega=0,-100 \mathrm{~cm}^{-1}$ and $-500 \mathrm{~cm}^{-1}$ with $T=4.2 \mathrm{~K}$ and a molecular field of $H_{M}=30 \mathrm{kOe}$.

are antiparallel, so that one gets only three different hyperfine fields as observed in the $\mathrm{Fe}-\mathrm{Cl}-$ and $\mathrm{Fe}-\mathrm{Br}$ boracite. The hyperfine fields in figure 7 show two pronounced minima at $\kappa=-10$ to -20 and $\kappa=5$ to 10 . The maximum in between for small $\kappa$ results from the fact that the orbital contribution has a large positive contribution compared with the negative Fermi contact field.

Increasing $\kappa$ the orbital contribution decreases and the hyperfine field becomes negative. From comparison of the ratios of the absolute values and of the angles $\theta, \varphi$ of the hyperfine fields with the measured parameters for the $\mathrm{Fe}-\mathrm{Cl}$ - and $\mathrm{Fe}-\mathrm{Br}$-boracite one obtains best agreement with $\kappa>5$ and $\omega<-100 \mathrm{~cm}^{-1}$, which implies that the E term is the electronic ground state.

All spin configurations which come from rotation around the c-axis (Fig. 6) with angles smaller $90^{\circ}$ lead to more than three lattice sites, which is equivalent with a strong tilting of the spins in the antiferromagnetic sublattices. This could be the reason for the measured four hyperfine fields in the Fe-I-boracite.

A further restriction for the possible values of $\kappa$

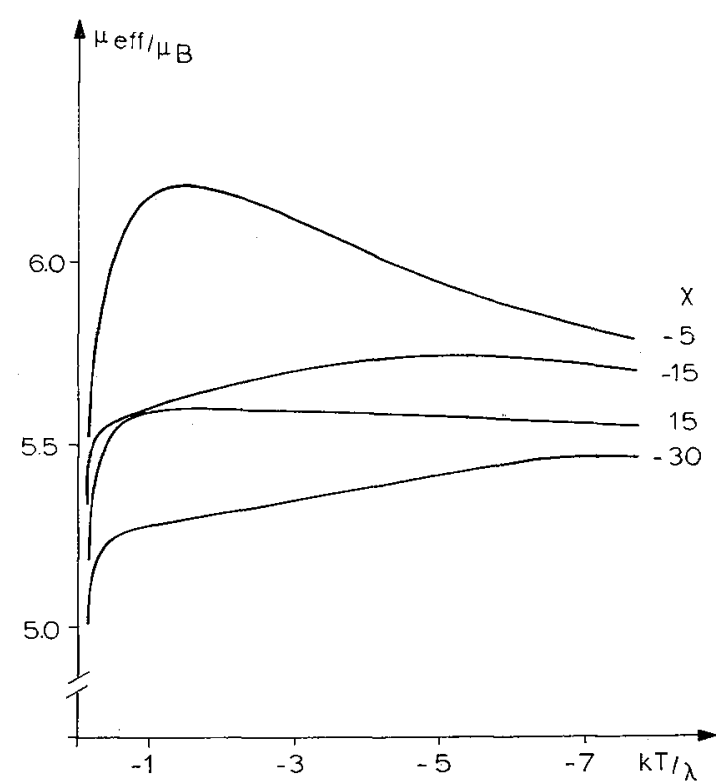

FIG. 8. $-\mu_{\text {eff }}$ as function of $k T / \lambda$ for different parameter values $\kappa_{.} \omega=-100 \mathrm{~cm}^{-1} ; \mu_{\mathrm{efP}}$ in units of $\mu_{\mathrm{B}}$. 
can be obtained from susceptibility measurements. Replacing the interaction of the spins with the molecular field in eq. (5) by the interaction with an external magnetic field $\mathbf{H}_{e}$, one gets

$$
\mathfrak{H}_{\mathrm{eff}}^{\prime \prime}=\mathscr{H}_{\mathrm{eff}}+\mu_{\mathrm{B}} \mathbf{H}_{\mathrm{e}}(\mathbf{L}+2 \mathbf{S}) .
$$

The susceptibility $\chi_{i}(i=\xi, \eta, \zeta)$ can be calculated according to van Vleck [19] by diagonalization of $\mathcal{H}_{\text {eff }}^{\prime \prime}$ with the same basis vectors set as for $\mathcal{H}_{\text {eff. }}^{\prime}$. From this one obtains the effective magnetic moment for the Fe-ion as function of temperature using the following relation :

$$
\mu_{\mathrm{eff}}=\sqrt{\frac{\bar{\chi} 3 k T}{N}} .
$$

Here $\bar{\chi}=\frac{1}{3}\left(\chi_{\xi}+\chi_{\eta}+\chi_{\xi}\right)$ and $N$ is the number of $\mathrm{Fe}$-ions in one magnetically ordered sublattice. In figure 8 we have plotted $\mu_{\mathrm{eff}}$ as function of $k T / \lambda$ for $\omega=-100 \mathrm{~cm}^{-1}$ and several parameters $\kappa$. The measurement of the susceptibility by $H$. Schmid et al. [6] shows that $\mu_{\mathrm{eff}}$ is independent of temperature in a large temperature scale. This is in accordance with the calculation for $\kappa=15$, which also gives good agreement with the measured absolute value of $\mu_{\text {eff }}=5.6 \mu_{\mathrm{B}}$.

The calculations show that the number and different sizes of the hyperfine fields in the Fe-boracites can be explained by the strong magnetic anisotropy and by taking into account the measured spin structure.

Acknowledgments. - The authors would like to thank Prof. Dr. E. Kankeleit for his continuous support and helpful discussions, Dr. G. Keller and Dr. H. Schmid for kindly providing us with the boracite crystals and Dr. J. M. Trooster for giving us his results prior to publication.

\section{References}

[1] BECKER, W. J. and WILL, G., Z. Kristallogr, 131 (1970) 139.

[2] Kobayashi, J., Sato, Y. and Schmid, H., Phys. Stat. Sol. 10 (1972) 259.

[3] Dowty, E. and Clark, J. R., Solid State Commun. 10 (1972) 543.

[4] Trooster, J. M., Dissertation 1967, Nijmwegen.

[5] SCHMID, H., RizDer, H. and AsCHER, E., Solid State Commun. 3 (1965) 327.

[6] Quezer, G. and Schmid, H., Solid State Commun. 6 (1968) 447.

[7] Kalvius, G. M. and Kankeleit, E., Mössbauer Spectroscopy and its Applications, International Atomic Energy Agency, Vienna (1972).

[8] KanKeleit, E., Mössbauer effect Methodology 1 (Plenum Press) $1965,47$.

[9] Schmro, H., J. Phys. Chem. Solids 26 (1965) 973.

[10] Stevens, J. G. and Stevens, V. E., Mössbauer Effect Data Index (Adam Hilger, London) 1970.
[11] Jagannathan, R., Trooster, J. M. and Viegers, M. P. A., Preprint (1973), submitted to the International Journal of Magnetism.

[12] Ingalls, R., Phys. Rev. 133A (1964) 787.

[13] Pisarev, R. V., Druzhinin, V. V., Nesterova, N. N., Prochorova, S. D. and Andreeva, G. T., Phys. Stat. Sol. 40 (1970) 503.

[14] DoRmanN, E., Dissertation 1969, Darmstadt.

[15] Sternheimer, R. M., Phys. Rev. 146 (1966) 140.

[16] Wartburg, v., W., Phys. Stat. Sol. 21 (1974) 557.

[17] Abragam, A. and Bleaney, B., Electronic Paramagnetic Resonance of Transition Ions (Oxford at the Clarendon Press) 1970.

[18] Freemann, A. J. and Frankel, R. B., Hyperfine Interactions (Acadenic Press, New York, London) 1967.

[19] Vleck van, J. H., The Theory of Electric and Magnetic Susceptibilities (Oxford University Press) 1932. 